\title{
CKD risk factors are associated with increased single-nephron GFR
}

The lack of an increase in singlenephron GFR with normal healthy ageing supports an age-based approach to defining CKD
Current assessment of kidney function in clinical practice relies on total glomerular filtration rate (GFR). Nephron number varies considerably between individuals, however, and nephron loss might not result in a change in total GFR as the remaining nephrons might increase their filtration rates to compensate. Now, researcher Andrew Rule and colleagues have developed a method to determine GFR at the level of the single nephron.

"Prior studies on single-nephron GFR have largely been in rodents as the methods used were invasive and destructive to the kidney. We needed a new method that could be applied to living humans in a safe and ethical manner," says Rule. "Living kidney donors were an ideal study population because all the data and tissue biopsy samples that we needed to determine single-nephron GFR were already available through their routine clinical care in several large kidney transplant programmes."

To calculate single-nephron GFR, Rule and colleagues used a two-step method. First they multiplied the glomerular density of the kidney biopsy sample taken at the time of kidney donation by the total renal cortical volume obtained from the CT scan to obtain the total nephron number. They then divided the total measured GFR determined using iothalamate clearance by the total number of nephrons to obtain the average single-nephron GFR.

The researchers report substantial variation in nephron number (mean of $860,000 \pm 370,000$ nephrons per kidney) among their study population of 1,388 healthy kidney donors. The mean single-nephron GFR was
$80 \pm 40 \mathrm{nl}$ per min and showed little variation with age, sex or height $\leq 190 \mathrm{~cm}$.

"The lack of an increase in single-nephron GFR with normal healthy ageing supports an agebased approach to defining chronic kidney disease (CKD)," says Rule. "Age is not currently a consideration for identifying patients with CKD."



Risk factors for CKD such as obesity and a family history of endstage renal disease (ESRD), as well as certain biopsy findings such as larger nephrons and a greater level of glomerulosclerosis and arteriosclerosis than would be expected for age, were associated with increased single-nephron GFR, as was height $>190 \mathrm{~cm}$. "As people age they lose nephrons owing to nephrosclerosis," explains Rule. "However, the remaining nephrons do not compensate by increasing single-nephron GFR unless the nephrosclerosis exceeds the level expected for age."

The study also enabled the researchers to determine which aspects of kidney function reflect the number of nephrons and which aspects reflect single-nephron GFR. "CKD risk factors can relate to the underlying contributors to kidney function in very different ways," says Rule. "Obesity is associated with higher single-nephron GFR but not with a change in nephron number, leading to an increased total GFR, whereas family history of ESRD is associated with lower nephron number but a compensatory increase in single-nephron GFR, such that total GFR is unchanged. By contrast, uric acid levels are associated with lower nephron number but are not associated with single-nephron GFR, leading to a lower total GFR."

Rule acknowledges that the need for a kidney biopsy sample to determine glomerular density currently limits the clinical applicability of single-nephron GFR. The researchers are now investigating whether new imaging technologies can be used to determine nephron number and enable single-nephron GFR to be calculated without the need for an invasive procedure.

Ellen F. Carney

ORIGINAL ARTICLE Denic, A. et al. Single-

nephron glomerular filtration rate in healthy adults. N. Engl.J. Med. 376, 2349-2357 (2017) 